\title{
A modified car-following model with variable safety distance and its feedback control research
}

\author{
Ya-Zhou ZHENG ${ }^{1, a}$, Cai-Hong YE ${ }^{1, b}$, Hong-Xia GE ${ }^{1,2,3, c}$, Zan ZHANG ${ }^{4, *}$
}

1. Ningbo University, Ningbo, 315211, China

2. Jiangsu Key Laboratory of Urban ITS, Southeast University, Nanjing 210096, China

3. National Traffic Management Engineering and Technology Research Centre

Ningbo University Sub-centre, Ningbo 315211, China

4. School of Science, East China University of Science and Technology, Shanghai 200237, China

a) zhengyazhoumath@163.com; b) yecaihong@nbu.edu.cn; c) gehongxia@nbu.edu.cn

*Corresponding author :zhangzan@ecust.edu.cn

Keywords: traffic flow; stability condition; feedback control method

\begin{abstract}
In this study, a modified car-following model is proposed to suppress the traffic jams. The optimal velocity (OV) function is extended by introducing variable safety headway distance. A comprehensive control scheme is constructed according to the feedback control theory. The stability condition for the modified model is obtained and the numerical simulation is carried out to illustrate the advantage of our model with the new control signal, and the results are consistent with the theoretical analysis.
\end{abstract}

\section{Introduction}

Over the last decades, traffic flow has gained more and more interests of many scholars and scientists, and traffic congestion has been a serious problem in the modern city traffic. In order to solve the increasingly serious traffic problems, research has been done on investigating the properties of traffic jams as much as possible, and obtained many significant results [1-7].

However, in expressway or highway with higher speed and larger headway, the models mentioned cannot be used to precisely reflect the real traffic phenomenon. In this case, the hyperbolic tangent part $(\tanh ()$.$) in OVM remains as a constant. That is to say, the following vehicle$ gets little or no influence despite the headway difference $\Delta x_{n}(t)$ is changing. So it is necessary to extend the typical safety headway distance parameter.

Up to the present time, there has been a limited amount of research work concerning carfollowing model from the viewpoint of control methods [8-11]. And even fewer studies in the area have been conducted with considering variable safety headway distance(VSHD). In this paper, a new optimal velocity (OV) function with considering high speed following vehicle on expressway or highway is constructed by introducing variable safety headway distance.

\section{IMPROVED Car-following model}

To investigate the effect of high speed and large headway of vehicles in expressway or highway, a new car-following model is presented as follows:

$$
\frac{d^{2} x_{n}(t)}{d t^{2}}=a\left[V_{\text {new }}^{o p}\left(\Delta x_{n}(t), v_{n}(t)\right)-v_{n}(t)\right]
$$

where $\Delta x_{n}(t)=x_{n+1}(t)-x_{n}(t)$ is the headway difference between the $n$ - $t$ considering vehicle and the preceding one; $x_{n}(t)$ is the $n$ - th vehicle's position; $a$ is the sensitivity of driver and is the inverse of delay time; $V_{\text {new }}^{\text {op }}\left(\Delta x_{n}(t), v_{n}(t)\right)$ is the new optimal velocity function, which determines by the 
headway difference $\Delta x_{n}(t)$ and the variable safety headway distance. The new OV function can be expressed as:

$$
\begin{aligned}
V_{\text {new }}^{\text {op }}\left(\square x_{n}(t)\right)=\frac{v_{\max }}{2}\left[\tanh \left(\square x_{n}(t)-h_{c}\right)+\tanh \left(h_{c}\right)\right](2) & \\
& h_{v}=d v_{n}(t) T_{s}+h_{c}
\end{aligned}
$$

where $v_{\max }$ is the maximum speed of all the following vehicles, $h_{c}$ is the typical safety headway distance; $T_{s}$ is the time step unit and $d$ is the reaction coefficient for $v_{n}(t)$. In real traffic flow, the driver does not care about the specific value of the safety headway distance, unless the numerical value means traffic accident or danger. So the physical meaning of $h_{v}$ is the acceptable safety headway distance of a driver and it is variable and related with velocity of the considered vehicle.

The dynamical equation is rewritten as follows:

$$
\left\{\begin{array}{l}
\frac{d v_{n}(t)}{d t}=a\left[V_{\text {new }}^{\text {op }}\left(y_{n}(t), v_{n}(t)\right)-v_{n}(t)\right] \\
\frac{d y_{n}(t)}{d t}=v_{n+1}(t)-v_{n}(t)
\end{array}\right.
$$

Where $y_{n}(t)=\Delta x_{n}(t)$, and we suppose that the leading vehicle runs at a constantly velocity $v_{0}$. Then, the steady state of the following vehicles are given by

$$
\left[v_{n}^{*}(t), y_{n}^{*}(t)\right]^{T}=\left[v_{0}, V_{\text {new }}^{\text {op }}{ }^{-1}\left(v_{0}\right)\right]^{T}
$$

According to the control theories, we can obtain the stability condition for the system (4), which is

$$
a \geq 2 \Lambda_{0}=\left.2 \frac{d V_{\text {new }}^{o p}\left(y_{n}(t), v_{n}(t)\right)}{d y_{n}(t)}\right|_{y_{n}(t)=V_{\text {new }}^{o p^{-1}}\left(v_{0}\right)}
$$

\section{Feedback Control scheme}

Considering the positive and negative velocity differences and optimal velocity differences into account, in the meantime, in order to avoid collision, the difference between the safety headway and the headway is considered. The new feedback control signal $u_{n}(t)$ including all these factors is added into system (1), so we have

$\frac{d^{2} x_{n}(t)}{d t^{2}}=a\left[V_{n e w}^{o p}\left(\Delta x_{n}(t), v_{n}(t)\right)-v_{n}(t)\right]+u_{n}(t)$

$u_{n}(t)=\kappa\left[\Delta v_{n}(t)+\Delta V_{\text {new }}^{\text {op }}(t)\right]-\lambda^{2} H\left(y_{n}(t)-h_{v}\right)\left(h_{v}-y_{n}(t)\right) \cdot(8)$

where $\Delta V_{\text {new }}^{\text {op }}(t)=\left[V_{\text {new }}^{\text {op }}\left(y_{n+1}(t), v_{n+1}(t)\right)-V_{\text {new }}^{o p}\left(y_{n}(t), v_{n}(t)\right)\right\rfloor$

and $\Delta v_{n}(t)=v_{n+1}(t)-v_{n}(t) . \kappa(\kappa>0)$ is the reaction coefficient for the relative optimal velocity $\Delta V_{\text {new }}^{\text {op }}(t)$ and the relative velocity $\Delta v_{n}(t), \lambda$ is another reaction coefficient for the $H\left(y_{n}(t)-h_{v}\right)\left(h_{v}-y_{n}(t)\right)$. And function $H($.$) is defined as$

$$
H\left(y_{n}(t)-h_{v}\right)= \begin{cases}0, & y_{n}(t)-h_{v}>0 \\ 1, & y_{n}(t)-h_{v} \leq 0\end{cases}
$$

As $y_{n}(t)-h_{v} \leq 0$, the feedback control signal $u_{n}(t)$ could simplify further. Then, our feedback control signal $u_{n}(t)$ is

$u_{n}(t)=\kappa\left[\Delta v_{n}(t)+\Delta V_{\text {new }}^{o p}(t)\right]-\lambda^{2}\left(h_{v}-y_{n}(t)\right)$

can be expressed as

Under this condition, the dynamical Eq.(7) 


$$
\left\{\begin{array}{l}
\frac{d v_{n}(t)}{d t}=a\left[V_{\text {new }}^{\text {op }}\left(y_{n}(t), v_{n}(t)\right)-v_{n}(t)\right]+\kappa\left[\left(v_{n+1}(t)-v_{n}(t)\right)\right]+ \\
\kappa\left[V_{\text {new }}^{\text {op }}\left(y_{n+1}(t), v_{n+1}(t)\right)-V_{\text {new }}^{\text {op }}\left(y_{n}(t), v_{n}(t)\right)\right]-\lambda^{2}\left(h_{v}-y_{n}(t)\right) \\
\frac{d y_{n}(t)}{d t}=v_{n+1}(t)-v_{n}(t)
\end{array}\right.
$$

leading vehicle runs with a constant velocity $v_{0}$, the following vehicles have the steady state as Eq.(5). Hence, the traffic system (11) can be linearized at steady state (5), that is

$$
\begin{aligned}
& \left\{\begin{array}{l}
\frac{d \delta v_{n}(t)}{d t}=a\left[\delta y_{n}(t) \Lambda_{0}-\delta v_{n}(t)\right]+\kappa\left[\left(\delta v_{n+1}(t)-\delta v_{n}(t)\right)\right]+ \\
\kappa\left[\delta y_{n+1}(t) \Lambda_{1}-\delta y_{n}(t) \Lambda_{0}\right]+\lambda^{2} \delta y_{n}(t) \quad(12) \quad \text { where } \quad \delta v_{n}(t)=v_{n}(t)-v_{0} \quad, \\
\frac{d \delta y_{n}(t)}{d t}=\delta v_{n+1}(t)-\delta v_{n}(t)
\end{array}\right. \\
& \delta y_{n}(t)=y_{n}(t)-V_{n e w}^{o p^{-1}}\left(v_{0}\right), \Lambda_{1}=\left.\frac{d V_{n e w}^{o p}\left(y_{n+1}(t), v_{n+1}(t)\right)}{d y_{n+1}(t)}\right|_{y_{n+1}(t)=V_{n e w}^{o-1}\left(v_{0}\right)} .
\end{aligned}
$$

After the Laplace transform for Eq.(12), we can get the transfer function $G(s)$ as follows:

$G(s)=\frac{1}{p(s)}\left[\kappa s+a \Lambda_{0}-\kappa \Lambda_{0}+\lambda^{2} s \kappa \Lambda_{1}\right]$

$p(s)=s^{2}+(a+\kappa) s+(a-\kappa) \Lambda_{0}+\lambda^{2}$

(14) where $s$ is a complex variable.

In reality, traffic jams will never occur in the traffic flow system with condition that the characteristic function $p(s)_{\text {is stable and }}\|G(s)\|_{\infty} \leq 1$ according to the control theory. In fact, based on the Hurwitz stability criterion, we can get that $p(s)$ is stable. Further, consider $\|G(s)\|_{\infty} \leq 1$, we can achieve the sufficient condition by means of the analysis above, that is

$0 \leq \lambda \leq \sqrt{\frac{a^{2}+2 \kappa\left(a+\Lambda_{0}-\kappa \Lambda_{1}^{2}\right)-2 a \Lambda_{0}}{2}} \quad$ (15) where $a^{2}+2 \kappa\left(a+\Lambda_{0}-\kappa \Lambda_{1}^{2}\right)-2 a \Lambda_{0} \geq 0$.

\section{Numerical simulation}

In the simulations, the parameters for vehicles are set as $h_{c}=7.02 \mathrm{~m}, a=2 \mathrm{~s}^{-1}, v_{0}=20 \mathrm{~m} / \mathrm{s}$ and $T=0.1 \mathrm{~s}$. It is assumed that all vehicles have the same parameters. Particularly, in Fig.1, the parameters are set to be $\kappa=0.85$,

$d=0.3, v_{\max }=33.3 \mathrm{~m} / \mathrm{s}$. The initial condition is the steady state for the model, and the initial positions and speeds are set as

$x_{n}(0)=\sum y_{n}^{*}, y_{n}(0)=y_{n}^{*}(t), v_{n}(0)=v_{n}^{*}(t)$

$n=1,2,3, \ldots, N$. And $N=120$ is the total number of vehicles. We consider a case where the leading vehicle stops suddenly for $t=n T=100-103$. 


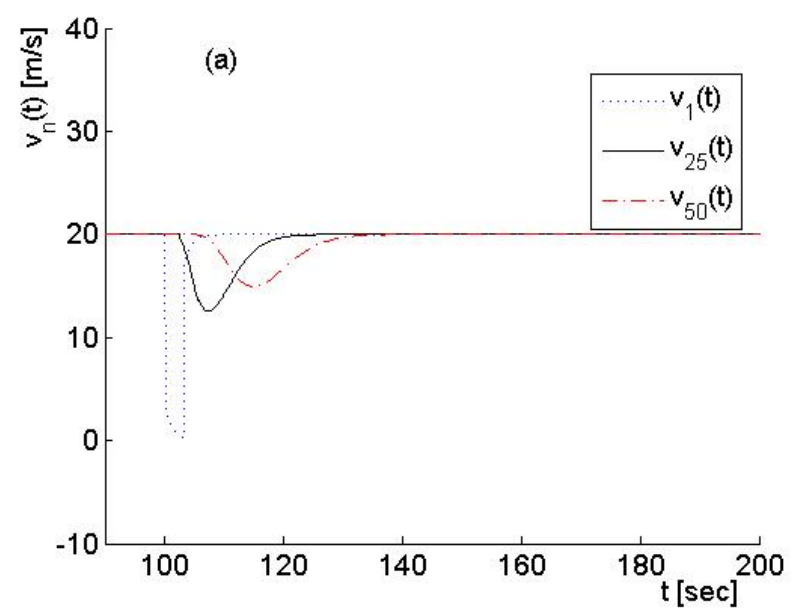

(a)

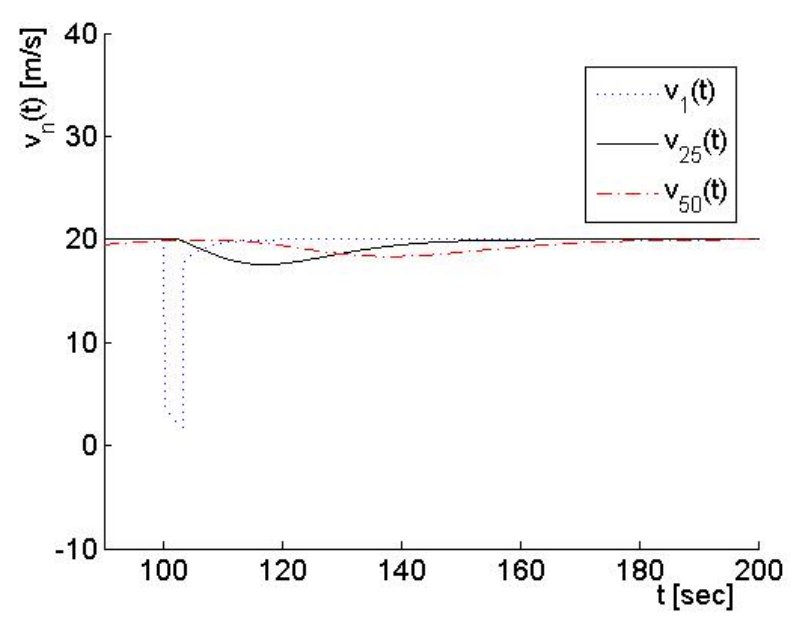

(b)

Fig. 1 Numerical simulations for the improved car-following model with (a) $\lambda=0.85$,(b) $\lambda=0.48$.

Fig.1 illustrates the speed-time patterns of the 1th, the 25th and the 50th vehicles with different parameter values of $\lambda$. It can be seen from Fig. 1 that with the control signal, when the reaction coefficient $\lambda$ decreases from 0.85 to 0.48 , the stability of the traffic system is strengthened. After further observation, we can obtained that vehicles can reach steady running state in relatively short time as the reaction coefficient $\lambda$ decreases. Moreover, the amplitude of the velocity for the 25th vehicle decreases gradually and the 50th vehicle runs more steadily.

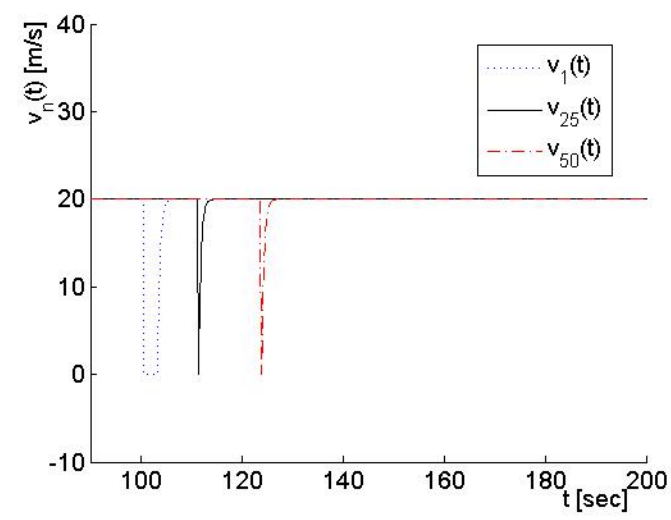

(a)

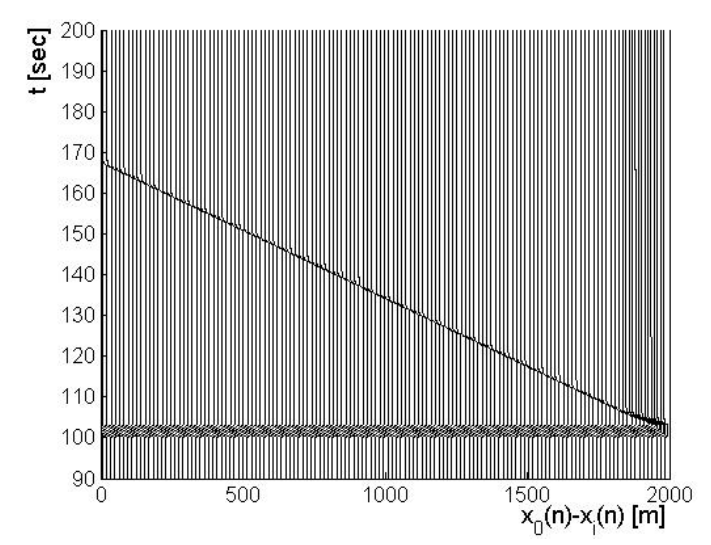

(b)

Fig. 2 (a) Space-time plot of the traffic system . (b) Temporal velocity behavior of the first, 25th and 50th vehicles. $\left(\kappa=0, \lambda=0, d=0, v_{\max }=20 \mathrm{~m} / \mathrm{s}\right)$. 
The simulation results in Figs.2-3 demonstrate the advantage of the improved car-following model. Although the maximum speed is larger in Fig.3 compared with Fig.2, we discover that vehicles can reach a more steady running state in comparatively short time, with the right parameters. The amplitude of the speed fluctuation for the 25th vehicle decreases and the 50th vehicle runs more smoothly. In other words, when we add a feedback control signal to system (1) with the new OV function, the traffic flow gets to a steady state quickly. We can conclude that the modified car-following model with new feedback control scheme is effective for suppressing the increasingly traffic congestion.

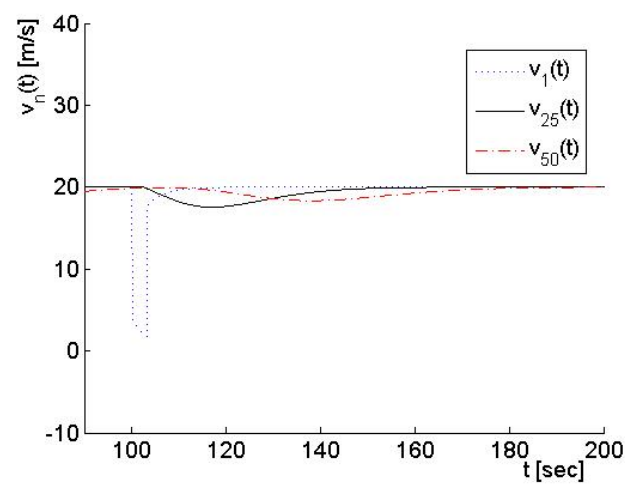

(a)

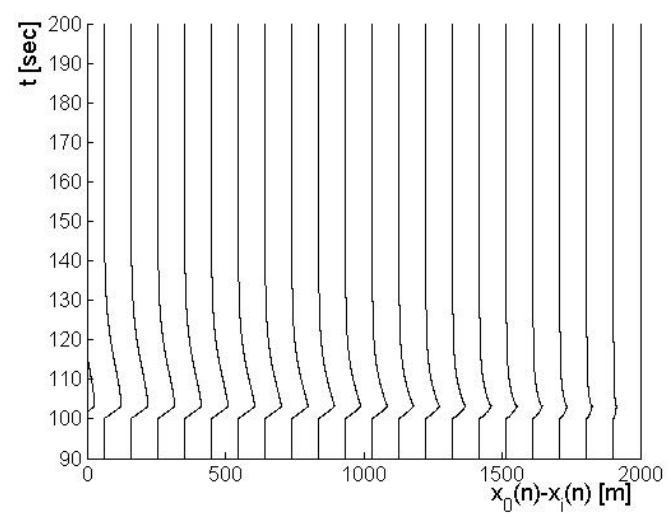

(b)

Fig. 3 (a) Space-time plot of the traffic system . (b) Temporal velocity behavior of the first, 25th and 50th vehicles. $\left(\kappa=75, \lambda=0.48, d=0.3, v_{\max }=33.3 \mathrm{~m} / \mathrm{s}\right)$.

\section{Conclusions}

In the study, an improved car-following model considering the variable safety headway distance based on the OVM is presented. Further, a new feedback control signal is added to OVM. The effect of some vital information (such as the relative velocity, relative optimal velocity and the difference between safety distance and headway) on the traffic current and the jamming transition has been investigated by use of the numerical and analytical methods. Moreover, the control method is adopted to derive the stability condition of the new model. Numerical simulations have been carried out and the results conform to the theoretical ones.

\section{Acknowledgments}

The project supported by the Fundamental Research Funds for the Central Universities(Grant No. 222201314049), the Science Foundation of Ningbo (Grant Nos. 2014A610022 and 2014A6111015), Humanities and Social Science Project of Ministry of Education of China (Grant No.14jyc630171), Soft Science Project of Zhejiang Province (Grant No.2014C35076) the Open Research Fund of Jiangsu Key laboratory of Urban ITS, Southeast University and the K. C. Wong Magna Fund in Ningbo University, China.

\section{References}

[1] B. S. Kerner, and H. Rehborn, "Experimental properties of complexity in traffic flow," Phys. Rev. E, 53: 1297, 1996.

[2] R. Jiang, Q. S. Wu and Z. J. Zhu, "A new continuum model for traffic flow and numerical tests,” Trans. Res. B. 36:405, 2002.

[3] Y. S. Qiang, J. W. Zhen, J. W. Du, Y. F. Liu, M. Wan and J. Wei, “Cellular automaton traffic fl ow model considering influence of accidents,” Acta Phys. Sin. 60: 060505, 2010.

[4] L. A. Pipes, “An Operational Analysis of Traffic Dynamics,” Journal of Applied Physics, 24(3): 274, 1953. 
[5] M. Bando, K. Hasebe, K. Nakanishi and A. Nakayama, "Analysis of optimal velocity model with explicit delay,” Phys. Rev. E. 58: 5429, 1998.

[6] R. Jiang, Q.S. Wu, Z.J. Zhu, "Full velocity difference model for a car-following theory,” Phys. Rev.E. 64: 017101, 2001.

[7] T. Q. Tang, Y. H. Wu, L. Caccetta and H. J. Huang, “A new car-following model with consideration of roadside memorial,” Phys.Lett. A. 375: 3845, 2011.

[8] H. X. Ge, S. Q. Dai, Y. Xue and L. Y. Dong, "Stabilization analysis and modified Korteweg-de Vries equation in a cooperative driving system,” Phys Rev. E, 71: 066119, 2005.

[9] K. Konishi, H. Kokame and K. Hirata, "Decentralized delayed-feedback control of an optimal velocity traffic model,” The European Physical Journal B, 15: 715, 2000.

[10] Y. Z. Zheng, P. J. Zheng , H. X. Ge, "An improved car-following model with considering lateral effect and its feedback control research,” Chin. Phys. B. 23(2): 020503, 2014.

[11] D. H. Sun, T. Zhou, W. N. Liu , L. J. Zheng, “A modified feedback controlled car-following model considering the comprehensive information of the nearest-neighbor leading car," Acta Phys Sin. 62: 170503, 2013 (In Chinese). 